\title{
Verification analysis for the reliable analytical multi-taper detector in next generation network
}

\author{
Hebat Allah O. Selim ${ }^{1}$, Ahmed Shaaban Dessouki ${ }^{2}$, Heba Y. M. Soliman ${ }^{3}$ \\ ${ }^{1}$ Department of Electrical and Engineering, Science Valley Academy, Egypt \\ ${ }^{2,3}$ Department of Electrical Engineering, Port Said University, Egypt
}

\section{Article Info \\ Article history: \\ Received Oct 30, 2019 \\ Revised Feb 21, 2020 \\ Accepted Mar 23, 2020}

\section{Keywords:}

Cognitive radio

Detectors

Estimators

Multitaper

Spectrum sensing

\begin{abstract}
Multi-taper method (MTM) acts as an effective detector of spectrum sensing in Cognitive radio networks. In this paper, an analytical study was proposed in which reliable, simple, and computationally efficient mathematical terms for the mean and variance of the probability density function (PDF) were derived using the MTM technique. The closed-form expressions for the probability of detection and false alarm for the MTM detector were obtained accordingly. The proposed analytical study was evaluated by intensive simulations using MATLAB. Different simulation techniques were proposed to verify the derived analysis. The existence of white Gaussian noise was assumed. Important aspects of spectrum detection in cognitive radio networks were included such as, receiver operating characteristics, detection rate versus signal to noise ratio (SNR), and the minimum desired sample points for a specific performance. A comparison was completed with the energy detection technique and all of the results suggested that the proposed paradigm is both credible and powerful under all the parameters considered in the simulation.
\end{abstract}

This is an open access article under the CC BY-SA license.

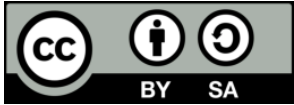

\section{Corresponding Author:}

Hebat Allah O. Selim,

Department of Electrical Engineering,

Science Valley Academy,

8 Road, Ahmed Orabi, El-Obour, Egypt.

Email: Heba.omar@sva.edu.eg

\section{INTRODUCTION}

As more technologies are transitioning to being completely wireless; both novel wireless applications, and services are expected to increase rapidly. As a result, the spectrum scarcity problem is worsening in certain bands. Cognitive radio (CR) which has been introduced by Mitola et al. [1] is a promising candidate to solve the spectrum deficiency issue by utilizing the radio spectrum in a more than an efficient manner. A reliable sensing technique is required to detect these resources, and to protect the primary user from interference.

The unpredictability of wireless signals [2-10] motivates the sensing algorithms to develop more methods to analyze different signal types. This is because the wireless signals often contain unknown parameters; such as: amplitude, phase, time delay, and frequency. These parameters must be first estimated before signal detection. Sensing algorithms are classified into two categories: parametric and non-parametric procedures. Parametric procedures are paradigm-based approaches, where some previous knowledge of the signal is known ahead of time. Using this information, a signal model can be assumed prior to the calculation of the power spectrum density [11, 12], such as matched filter and cyclostationary detection methods [13-15]. Despite the estimation being more accurate and precise; previous knowledge of the primary user's (PU's) signaling creates a major challenge for CR. 
Non-parametric, on the other hand, does not rely on a fixed set of parameters, since these parameters are varying over time. Under no assumptions about the physical process of a given data, the power is estimated from Fourier-based methods where no prior model is supposed. The conventional non-parametric methods such as periodogram detection [16] suffer from spectral leakage effects, resulting from bad bias, that often mask weak signals. The recent non-parametric techniques such as multi-taper [17] always compromises between the bias and the variance of the detected signals. In theory, MTM reduces the variance of the power estimates by obtaining the signal power from the sum of an orthogonal narrowband windows that have the same length. In other words, this method reduces estimation variance by obtaining multiple independent estimates from the same samples. This is done by employing a small set of windows rather than the unique data window or spectral taper.

The underlying concept of multi-taper detector is based on applying a set of windows sequences, which Thomson discussed in [18]. These sequences are known as discrete prolate Slepian wave functions (DPSWF). The most clarifying fact about these sequences is the orthogonal property, which means that, the DPSS will return the O/P as uncorrelated independent sequences, in addition to the different variance for each time sequence. Combining all these sequences concentrates the power in a main lobe and reduces it in the side lobes. Furthermore, there is an obvious improvement in the variance.

Most literature on this subject [19-22] provides the analytical formula of multi-taper based detector, which is useful to a particular extent in spectrum sensing; however, there is a need for simple analytical closed-from terms for numerical evaluations. In [19], an optimal detection is also proposed, and applied using actual HDTV data. However, the mean $(\mu)$ and variance $\left(\sigma^{2}\right)$ are calculated from complicated expressions that need to be done through a numerical method to calculate the threshold voltage.

In [20], an optimal detector for enhancing the sensing performance of multi-taper detector in CR networks is discussed. The analysis is based on Neyman-Pearson theorem, in which the ratio between the power spectrum density PSD for the signal affected by noise and PSD for noise only, has to be maximized through the likelihood ratio test (LRT). The natural logarithm is also used to smooth the estimated power. The mean and variance of the power spectrum density are derived from the smooth estimated power. The results show that the proposed detector is more accurate than the energy detector since there is a $48 \%$ increase in detection performance in comparison to the energy detector. In addition, the desired sample size $(\mathrm{N})$ of the proposed detector is reduced by $73.3 \%$. However, the building blocks of the mean and variance, are computationally demanding.

In [22], the estimated power is approximated from chi-square distribution to normal distribution for large sample size. The simple formula for the mean and variance are derived accordingly. Regarding to the derived analysis, the variance does not change as the number of tapers $(\mathrm{K})$ rises. It should be observed that the mean is a linear function of $(\mathrm{K})$, which implies that the mean proportionally increases with the number of tapers (K). Therefore, the derived formulas contradict the concept of MTM.

Considering the above limitations, a reliable, and a simple computation of a mathematical closed form expression of MTM detector is derived. Through an intensive analysis of different simulation parameters, the precision of the proposed paradigm is verified. The results demonstrate that the proposed paradigms outperform other detection systems, for example, it is $40 \%$ better than the energy detector. Also, this system needs only $(\mathrm{K})$ times of sample to achieve the same detection accuracy. The results are also compared with a previous model of the same systems but a reliable one. It is noted from these results that the proposed paradigm achieved the same detection accuracy, using the same sample size, but at simple computation of mathematical expressions.

The remaining parts of the paper are organized as follows: Section 2 describes the MTM spectrum sensing method. In section 3, the proposed test statistic and correction factor is discussed. Finally, section 4 shows the simulation and discussion results. In section 5, the conclusion of the paper is presented.

\section{THE PROPOSED MULTI-TAPER PARADIGM}

A non- stationary signal generated from a random statistical process is considered. At the CR receiver, the signal is sampled to get the finite discrete sample $\mathrm{Xn} ; \mathrm{n}=0 ; 1: \mathrm{N}-1$, where $\mathrm{N}$ is the sample number. Then, Xn is multiplied by a number of discrete Slepian sequences $\mathrm{H}_{\mathrm{n}}{ }^{\mathrm{k}}(\mathrm{N}, \mathrm{W})$ of length $\mathrm{N}$ which are represented by orthogonal tapers to concentrate the energy of the received PU signal in the interval between $(-\mathrm{W}, \mathrm{W})$. The time to bandwidth product is $\mathrm{NW}$ and the total tapers is $\mathrm{K}=2 \mathrm{NW}-1$ with their associated Eigen values of the $\mathrm{k}^{\text {th }}$ taper are $\lambda_{\mathrm{k}}$. The products are, then, applied to FFT to get the power concentrated in a chosen bandwidth $(-\mathrm{W}, \mathrm{W})$, as shown in Figure 1. The dot product of the finite discrete sample sequence $\mathrm{Xn}$ and the $\mathrm{K}$ orthogonal tapers produces $\mathrm{K}$ different Eigen spectrums that are determined as: 
$Y_{k}\left(f_{i}\right)=\sum_{t=0}^{N-1} H_{t}^{k}(N, W) X_{t} e^{-2 j \pi f t}$

where fi are frequency bins and $\mathrm{k}=0 ; 1 ; \ldots ; \mathrm{K}-1$.

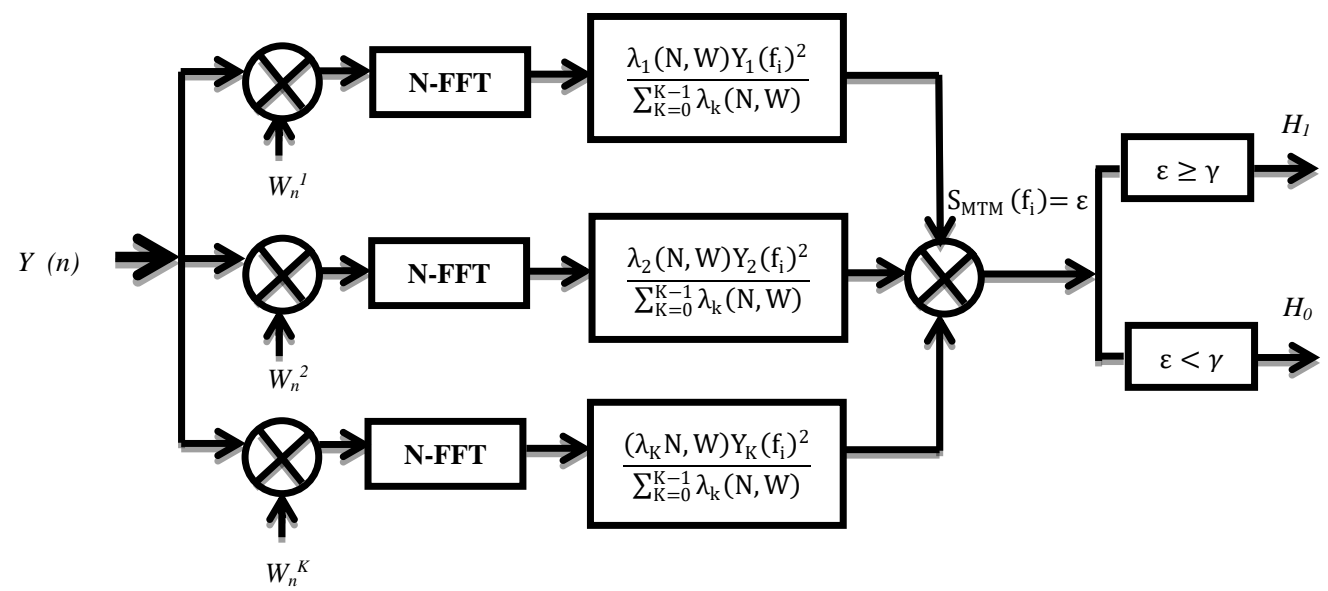

Figure 1. Multi-taper system model

According to Thomson equation [18], the estimated energy, which exhibits the least variance and sidelobe leakage, is obtained by averaging all of these sequences by their weighting Eigen values as:

$$
\mathrm{S}_{\mathrm{MTM}}\left(\mathrm{f}_{\mathrm{i}}\right)=\frac{\sum_{\mathrm{K}=0}^{\mathrm{K}-1} \lambda_{\mathrm{k}}(\mathrm{N}, \mathrm{W}) \mathrm{Y}_{\mathrm{k}}\left(\mathrm{f}_{\mathrm{i}}\right)^{2}}{\sum_{\mathrm{K}=0}^{\mathrm{K}-1} \lambda_{\mathrm{k}}(\mathrm{N}, \mathrm{W})}
$$

which can be written as:

$$
\mathrm{S}_{\mathrm{MTM}}\left(\mathrm{f}_{\mathrm{i}}\right)=\sum_{\mathrm{k}=0}^{\mathrm{K}-1} \mathrm{a}_{\mathrm{k}}(\mathrm{N}, \mathrm{W})\left|\mathrm{Y}_{\mathrm{k}}\left(\mathrm{f}_{\mathrm{i}}\right)\right|^{2}
$$

where the weights ak are defined as:

$$
\mathrm{a}_{\mathrm{k}}=\frac{\lambda_{\mathrm{k}}}{\sum_{\mathrm{k}=0}^{\mathrm{K}-1} \lambda_{\mathrm{k}}(\mathrm{N}, \mathrm{W})}
$$

The energy detection method, on the other hand, gives the power spectrum density (PSD) estimation as:

$$
S_{E D}\left(f_{i}\right)=\frac{1}{N} \sum_{t=0}^{N-1}\left|X_{t} e^{-2 j \pi f t}\right|^{2}
$$

Following the hypothesis model declared in (6) to (9), the MTM detector could be compared with other systems.

$$
\begin{array}{ll}
\mathrm{Y}(\mathrm{t})=\mathrm{n}(\mathrm{t}), & 0<t \leq T: \mathrm{H}_{0} \\
\mathrm{Y}(\mathrm{t})=\mathrm{S}(\mathrm{t})+\mathrm{n}(\mathrm{t}), & 0<t \leq T: \mathrm{H}_{1} \\
\mathrm{P}_{\mathrm{f}}=\mathrm{P}_{\mathrm{r}}(\varepsilon>\gamma) \mathrm{H}_{0} & \\
\mathrm{P}_{\mathrm{d}}=\mathrm{P}_{\mathrm{r}}(\varepsilon \geq \gamma) \mathrm{H}_{1} &
\end{array}
$$

Where $\mathrm{Y}(\mathrm{t})$ is the received signal, $\mathrm{S}(\mathrm{t})$ is the transmitted signal, $\mathrm{n}(\mathrm{t})$ is white noise, $\varepsilon$ is the test statistic and $\gamma$ is the threshold voltage. $\mathrm{H}_{0}$ denotes that primary user is absent while it is actually present, and $\mathrm{H}_{1}$ denotes that primary user is existing. 
In the proposed paradigm, the power distribution of the received signal is approximated to normal distribution at higher values of $\mathrm{N}$. According to the Neyman-Pearson criterion [23], $\mathrm{P}_{\mathrm{d}}$ and $\mathrm{P}_{\mathrm{f}}$ are expressed as:

$$
\begin{aligned}
& P_{d}=Q\left(\frac{\gamma-\mu / H_{1}}{\sqrt{\sigma^{2} / H_{1}}}\right) \\
& P_{f}=Q\left(\frac{\gamma-\mu / H_{0}}{\sqrt{\sigma^{2} / H_{0}}}\right)
\end{aligned}
$$

where $\mu / \mathrm{H}_{1}$ and $\sigma^{2} / \mathrm{H}_{1}$ are the mean and the variance of the received signal power under $\mathrm{H}_{1}$ hypothesis, respectively. $\mu / \mathrm{H}_{0}$ and $\sigma^{2} / \mathrm{H}_{0}$ are the mean and the variance of the received signal power under $\mathrm{H}_{0}$ hypothesis, respectively.

Using statistical tools for independent random variables, we calculate the mean and the variance considering the MTM concepts from the beginning. Since we have a number K of independent random variables, the estimated density of spectrum, using $\mathrm{K}$ tapers, is a summation of Eigen-spectrum with weights $\lambda_{\mathrm{k}}$.

$$
E\left[\widetilde{S}_{\text {Estimated }}(f)\right]=\sum_{i=0}^{K-1} a_{i} \widetilde{S}_{i}(f)
$$

where $\widetilde{S}_{\mathrm{i}}(\mathrm{f})=\sum_{\mathrm{i}=0}^{\mathrm{K}-1} \mid\left.\mathrm{FFT}\left(\right.$ Signal ${ }^{\chi}$ Taper $\left._{\mathrm{i}}\right)\right|^{2}$. The variance is calculated as:

$$
\text { Variance }=\sum_{\mathrm{i}=0}^{\mathrm{K}-1} \mathrm{a}_{\mathrm{i}}^{2} \operatorname{Var}\left[\widetilde{\mathrm{S}}_{\mathrm{i}}(\mathrm{f})\right]+2 \sum_{\mathrm{i} \& j=0}^{\mathrm{k}} \mathrm{a}_{\mathrm{i}} \mathrm{a}_{\mathrm{j}} \operatorname{Cov}\left[\widetilde{\mathrm{S}}_{\mathrm{i}}(\mathrm{f}) \widetilde{\mathrm{S}}_{\mathrm{j}}(\mathrm{f})\right]
$$

where covariance $(\mathrm{Cov})$ for $\mathrm{i} \neq \mathrm{j}$ is calculated by:

$$
\operatorname{Cov}\left[\widetilde{S}_{i}(f) \widetilde{S}_{j}(f)\right]=\rho \sigma_{i} \sigma_{j}
$$

where $\rho$ is the correlation coefficient. For $\mathrm{H}_{0}$ hypothesis where noise only exists, the mean of MTM $\left(\mu_{\mathrm{MTM}} / \mathrm{H}_{0}\right)$ and the variance $\sigma^{2}{ }_{\text {MTM }}$ can be calculated as:

$$
\begin{aligned}
& \mu_{\mathrm{MTM}} \backslash \mathrm{H}_{0}=\left(\frac{\lambda_{1}}{\sum \mathrm{k} \lambda_{\mathrm{k}}}\right) \mathrm{E}\left[\tilde{\mathrm{S}}(\mathrm{f})_{1}\right]+\left(\frac{\lambda_{2}}{\sum \mathrm{k} \lambda_{\mathrm{k}}}\right) \mathrm{E}\left[\tilde{\mathrm{S}}(\mathrm{f})_{2}\right] \ldots \ldots \ldots+\left(\frac{\lambda_{\mathrm{K}}}{\sum \mathrm{k} \lambda_{\mathrm{k}}}\right) \mathrm{E}\left[\tilde{\mathrm{S}}(\mathrm{f})_{\mathrm{K}}\right] \\
& =\left(\frac{\lambda_{1}}{\sum \mathrm{k} \lambda_{\mathrm{k}}}\right) \sigma_{\mathrm{w}}^{2}+\left(\frac{\lambda_{2}}{\sum \mathrm{k} \lambda_{\mathrm{k}}}\right) \sigma_{\mathrm{w}}^{2} \ldots \ldots \ldots+\left(\frac{\lambda_{\mathrm{K}}}{\sum \mathrm{k} \lambda_{\mathrm{k}}}\right) \sigma_{\mathrm{w}}^{2} \\
& =\left(\frac{\sigma_{\mathrm{w}}^{2}}{\sum \mathrm{k} \lambda_{\mathrm{k}}}\right)\left(\lambda_{1}+\lambda_{2} \ldots \ldots \ldots+\lambda_{\mathrm{k}}\right)=\sigma_{\mathrm{w}}^{2} \\
& \sigma_{\text {MTM }}^{2} / \mathrm{H}_{0}=\left(\frac{\lambda_{1}^{2}}{\left(\sum \mathrm{k} \lambda_{\mathrm{k}}\right)^{2}}\right) \times\left(\frac{2 \sigma_{\mathrm{w}}^{4}}{\mathrm{~N}}\right)+\left(\frac{\lambda_{2}^{2}}{\left(\sum \mathrm{k} \lambda_{\mathrm{k}}\right)^{2}}\right) \times\left(\frac{2 \sigma_{\mathrm{w}}^{4}}{\mathrm{~N}}\right) \ldots \ldots \ldots+\left(\frac{\lambda_{\mathrm{k}}^{2}}{\left(\sum \mathrm{k} \lambda_{\mathrm{k}}\right)^{2}}\right) \times\left(\frac{2 \sigma_{\mathrm{w}}^{4}}{\mathrm{~N}}\right) \\
& +2 \rho\left\{\left[\left(\frac{\lambda_{1} \lambda_{2}}{\left(\sum \mathrm{k} \lambda_{\mathrm{k}}\right)^{2}}\right) \times\left(\frac{2 \sigma_{\mathrm{w}}^{4}}{\mathrm{~N}}\right)\right]+\left[\left(\frac{\lambda_{1} \lambda_{3}}{\left(\sum \mathrm{k} \lambda_{\mathrm{k}}\right)^{2}}\right) \times\left(\frac{2 \sigma_{\mathrm{w}}^{4}}{\mathrm{~N}}\right)\right]+\cdots\right. \\
& +\left[\left(\frac{\lambda_{1} \lambda_{\mathrm{k}}}{\left(\sum \mathrm{k} \lambda_{\mathrm{k}}\right)^{2}}\right) \times\left(\frac{2 \sigma_{\mathrm{w}}^{4}}{\mathrm{~N}}\right)\right]+\left[\left(\frac{\lambda_{2} \lambda_{3}}{\left(\sum \mathrm{k} \lambda_{\mathrm{k}}\right)^{2}}\right) \times\left(\frac{2 \sigma_{\mathrm{w}}^{4}}{\mathrm{~N}}\right)\right] \\
& \left.+\cdots,+\left[\left(\frac{\lambda_{2} \lambda_{\mathrm{k}}}{\left(\sum \mathrm{k} \lambda_{\mathrm{k}}\right)^{2}}\right) \times\left(\frac{2 \sigma_{\mathrm{w}}^{4}}{\mathrm{~N}}\right)\right]+\cdots .+\left[\left(\frac{\lambda_{\mathrm{k}} \lambda_{\mathrm{k}-1}}{\left(\sum \mathrm{k} \lambda_{\mathrm{k}}\right)^{2}}\right) \times\left(\frac{2 \sigma_{\mathrm{w}}^{4}}{\mathrm{~N}}\right)\right]\right\} \\
& =\left(\frac{2 \sigma_{\mathrm{w}}^{4}}{\left(\sum \mathrm{k} \lambda_{\mathrm{k}}\right)^{2} \mathrm{~N}}\right) \chi\left(\lambda_{1}^{2}+\lambda_{2}^{2} \ldots \ldots \ldots+\lambda_{\mathrm{K}}^{2}\right) \\
& +\left[\left(\frac{2 \rho * 2 \sigma_{\mathrm{w}}^{4}}{\left(\sum \mathrm{k} \lambda_{\mathrm{k}}\right)^{2} \mathrm{~N}}\right) \chi\left(\lambda_{1} \lambda_{2}+\lambda_{1} \lambda_{3} \ldots \ldots+\lambda_{1} \lambda_{\mathrm{k}} \ldots .+\lambda_{\mathrm{k}-1} \lambda_{\mathrm{k}}\right)\right]
\end{aligned}
$$


For independent uncorrelated tapers with $\rho=0$, and for first higher order tapers where power concentration $\approx 1$.

$$
\frac{\lambda_{1}^{2}+\lambda_{2}^{2}+\cdots+\lambda_{\mathrm{K}}^{2}}{\left(\sum \mathrm{k} \lambda_{\mathrm{k}}\right)^{2}} \simeq \frac{1}{\mathrm{~K}}
$$

and

$$
\sigma_{\mathrm{MTM}}^{2} \backslash \mathrm{H}_{0}=\left(\frac{2 \sigma_{\mathrm{w}}^{4}}{\mathrm{NK}}\right)
$$

For hypothesis $\mathrm{H}_{1}$ where signal and noise exist:

$$
\begin{aligned}
\mu_{\mathrm{MTM}} \backslash \mathrm{H}_{1} & =\left(\frac{\lambda_{1}}{\sum \mathrm{k} \lambda_{\mathrm{k}}}\right) \mathrm{E}\left[\widetilde{\mathrm{S}}(\mathrm{f})_{1}\right]+\left(\frac{\lambda_{2}}{\sum \mathrm{k} \lambda_{\mathrm{k}}}\right) \mathrm{E}\left[\widetilde{\mathrm{S}}(\mathrm{f})_{2}\right] \ldots \ldots \ldots+\left(\frac{\lambda_{\mathrm{K}}}{\sum \mathrm{k} \lambda_{\mathrm{k}}}\right) \mathrm{E}\left[\widetilde{\mathrm{S}}(\mathrm{f})_{\mathrm{K}}\right] \\
& =\left(\frac{\lambda_{1}}{\sum \mathrm{k} \lambda_{\mathrm{k}}}\right)\left(\mathrm{E}_{\mathrm{s}}+\sigma_{\mathrm{w}}^{2}\right)+\left(\frac{\lambda_{2}}{\sum \mathrm{k} \lambda_{\mathrm{k}}}\right)\left(\mathrm{E}_{\mathrm{s}}+\sigma_{\mathrm{w}}^{2}\right) \ldots+\left(\frac{\lambda_{\mathrm{K}}}{\sum \mathrm{k} \lambda_{\mathrm{k}}}\right)\left(\mathrm{E}_{\mathrm{s}}+\sigma_{\mathrm{w}}^{2}\right) \\
& =\left(\frac{\left(\mathrm{E}_{\mathrm{s}}+\sigma_{\mathrm{w}}^{2}\right)}{\sum \mathrm{k} \lambda_{\mathrm{k}}}\right)\left(\lambda_{1}+\lambda_{2} \ldots \ldots \ldots+\lambda_{\mathrm{k}}\right)=\left(\mathrm{E}_{\mathrm{s}}+{ }_{\mathrm{w}}^{2}\right)
\end{aligned}
$$

and variance,

$$
\begin{aligned}
\sigma_{\mathrm{MTM}}^{2} \backslash \mathrm{H}_{1}= & \left(\frac{2 \sigma_{\mathrm{w}}^{4}(\mathrm{SNR}+1)^{2}}{\left(\sum \mathrm{k} \lambda_{\mathrm{k}}\right)^{2} \mathrm{~N}}\right) \times\left(\lambda_{1}^{2}+\lambda_{2}^{2} \ldots \ldots \ldots+\lambda_{\mathrm{K}}^{2}\right) \\
& +\left[\left(\frac{2 \rho^{\chi} 2 \sigma_{\mathrm{w}}^{4}(\mathrm{SNR}+1)^{2}}{\left(\sum \mathrm{k} \lambda_{\mathrm{k}}\right)^{2} \mathrm{~N}}\right) \times\left(\lambda_{1} \lambda_{2}+\lambda_{1} \lambda_{3} \ldots \ldots .+\lambda_{1} \lambda_{\mathrm{k}} \ldots \ldots \ldots+\lambda_{\mathrm{k}-1} \lambda_{\mathrm{k}}\right)\right]
\end{aligned}
$$

For independent uncorrelated Tapers, $\rho=0$, and for first higher order tapers where power concentration $\approx 1$.

$$
\sigma_{\text {MTM }}^{2} \backslash H_{1}=\left(\frac{2 \sigma_{\mathrm{w}}^{4}(\mathrm{SNR}+1)^{2}}{\mathrm{NK}}\right)
$$

According to the Neyman-Pearson criteria and using (10) and (11), the $\mathrm{P}_{\mathrm{d}}$ and $\mathrm{P}_{\mathrm{f}}$ can be obtained as:

$$
\begin{aligned}
& \mathrm{P}_{\mathrm{d}}^{\mathrm{MTM}}=\mathrm{Q}\left(\frac{\gamma-\sigma_{\mathrm{w}}^{2}(\mathrm{SNR}+1)}{\sqrt{\left(\frac{2 \sigma_{\mathrm{w}}^{4}(\mathrm{SNR}+1)^{2}}{\mathrm{NK}}\right)}}\right) \\
& \mathrm{P}_{\mathrm{f}}^{\mathrm{MTM}}=\mathrm{Q}\left(\frac{\gamma-\sigma_{\mathrm{w}}^{2}}{\sqrt{\left(\frac{2 \sigma_{\mathrm{w}}^{4}}{\mathrm{NK}}\right)}}\right) \\
& \gamma=\mathrm{Q}^{-1}\left(\mathrm{P}_{\mathrm{f}}^{\mathrm{MTM}}\right)^{x} \sqrt{\left(\frac{2 \sigma_{\mathrm{w}}^{4}}{\mathrm{NK}}\right)}+\sigma_{\mathrm{w}}^{2}
\end{aligned}
$$

On the other hand the $\mathrm{P}_{\mathrm{d}}$ and $\mathrm{P}_{\mathrm{f}}$ for energy detection can be written as:

$$
\begin{aligned}
& \mathrm{P}_{\mathrm{d}}^{\mathrm{En}}=\mathrm{Q}\left(\frac{\gamma-\left(\mathrm{E}_{\mathrm{s}}+\sigma_{\mathrm{w}}^{2}\right)}{\sqrt{\left(\frac{2 \sigma_{\mathrm{w}}^{4}(\mathrm{SNR}+1)^{2}}{\mathrm{~N}}\right)}}\right) \\
& \mathrm{P}_{\mathrm{f}}^{\mathrm{En}}=\mathrm{Q}\left(\frac{\gamma-\sigma_{\mathrm{w}}^{2}}{\sqrt{\left(\frac{2 \sigma_{\mathrm{w}}^{4}}{\mathrm{~N}}\right)}}\right)
\end{aligned}
$$




\section{THE TEST STATISTIC AND THE PROPOSED CORRECTION FACTOR}

The test statistic $(\varepsilon)$ of MTM, where the sensing decision is executed by comparing it with threshold, is calculated by two equivalent methods. In the first method, the test statistic is estimated from the mean of spectrum sensing given by (2).

The test $\operatorname{statistic}(\varepsilon)=\operatorname{mean}\left(S_{M T M}\left(f_{i}\right)\right)$

And in the second method, the MATLAB [pxx, w] function is used and the test statistic is proposed as:

The test $\operatorname{statistic}(\varepsilon)=\operatorname{mean}\left(\pi^{\chi} \mathrm{Pxx}\right)$

The sensing decision which is done according to the decision rule given in (8) and (9) is very sensitive for any small error in the test statistic $(\varepsilon)$. This error leads to a deviation of simulation results from the theoretical one as shown in Figure 2 for different simulation parameters. The figure depicts the mean square error (MSE) deviation error between the theoretical and simulated Pd.

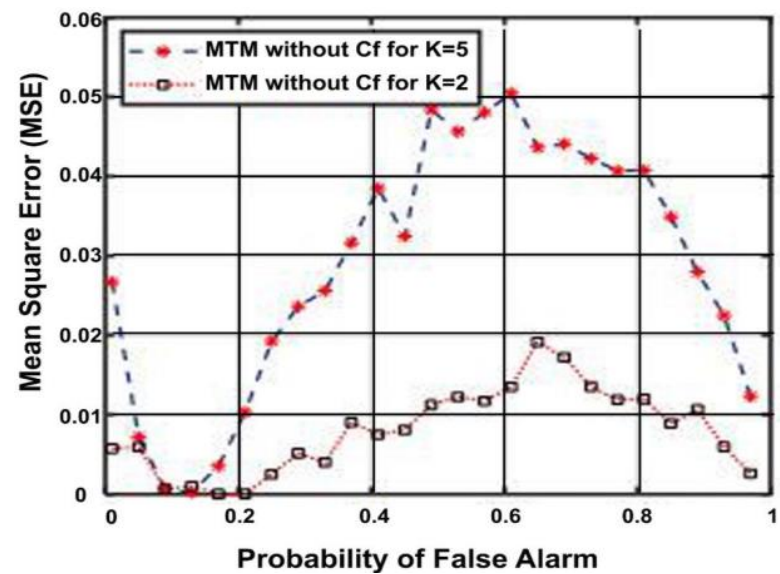

Figure 2. MSE deviation error between the theoretical and simulated $\mathrm{Pd}$ for $\mathrm{N}=512, \mathrm{SNR}=-15 \mathrm{~dB}$ with $\mathrm{K}=2,5$

From Figure 2, it is clear that the sign and the value of the deviation error depend on the simulation parameters. For example, it increases with the increase of $\mathrm{K}$ and it may be negative or positive for some ranges of $P_{f}$. Also, it increases (with negative sign) as $P_{f}$ decreases below about "0.2" because the values of $P_{d}$ are low in this region. After $\mathrm{P}_{\mathrm{f}}$ becomes greater than about " 0.2 ", it increases slightly and then decreases slowly as $\mathrm{P}_{\mathrm{f}}$ increases till being equal to about zero at $\mathrm{P}_{\mathrm{f}}=1$. The same analysis is done for the remaining parameters: SNR, K, and N. From the above observations and analysis, an empirical correction factor (Cf) is proposed as given in (29) to reduce this deviation error.

$$
C f=1+\{A+B+C\}
$$

where, $\quad A=\left\{\left[0.03{ }^{\chi} \mathrm{K} \times\left(\mathrm{P}_{\mathrm{f}}-0.2\right)+0.06^{\chi} \mathrm{P}_{\mathrm{f}} \times\left(1-\mathrm{P}_{\mathrm{f}}\right)+1.61^{\chi} \mathrm{SNR}-0.049\right]^{\chi}(1-1 / \mathrm{K})\right\}$

$B=\left\{\left(1.2 \mathrm{e}-5^{x} \mathrm{~N}\right)^{\chi}\left(1-\mathrm{P}_{\mathrm{f}}\right)^{\mathrm{x}}(1-15 \mathrm{e}-4 / \mathrm{SNR})\right\}$

$C=\left\{\left[0.175^{\chi} \mathrm{SNR}+0.025^{\chi} \mathrm{P}_{\mathrm{f}} \times\left(1.4^{\chi} \mathrm{SNR}-1\right)+5 \mathrm{e}-7^{\chi} \mathrm{P}_{\mathrm{f}}^{\chi}\left(1+1.5 \mathrm{e}-3^{\chi} \mathrm{N}\right)+5 \mathrm{e}-3\right]\right.$ $\chi(1-5 / K)\}$

To reduce the deviation error, the test statistic must be multiplied by "Cf" before making the decision.

Figure 3 displays the deviation error between the theoretical and simulated $\mathrm{P}_{\mathrm{d}}$. It is obvious that the deviation error has been addressed and the correction factor $(\mathrm{Cf})$ is working well for a wide range of simulation parameters except at very low values of $\mathrm{P}_{\mathrm{f}}\left(\mathrm{P}_{\mathrm{f}}<0.05\right)$. Figure 4 shows "Cf-1" \%, which is considered as the percentage deviation error of the simulated Test Statistic $(\varepsilon)$, versus $\mathrm{P}_{\mathrm{f}}$.

Figures 3 and 4, illustrate the impact of including the correction factor. From Figure 4, it is obvious that a short deviation error $\left(\mathrm{C}_{\mathrm{f}}-1\right) \%$ in the simulated test Statistic $(\varepsilon)$ results in a relatively large one between the theoretical and simulated $\mathrm{P}_{\mathrm{d}}$. For example, for $\mathrm{K}=5$, at $\mathrm{P}_{\mathrm{f}}=0.5$, the deviation error in the simulated $(\varepsilon)$ is about 5\%. From Figure 3, it results in about $22 \%$ deviation error in $\mathrm{P}_{\mathrm{d}}$ prediction. Significantly, after introducing the correction factor to the simulated test statistic $(\varepsilon)$, the deviation error reduced to zero as depicted in Figure 3. The effectiveness of proposed correction factor is verified in the next section. 


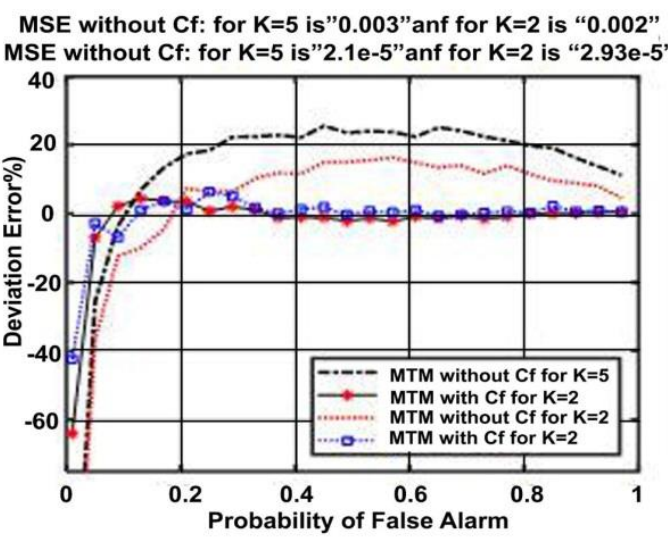

Figure 3. Deviation error \% between the theoretical and simulated Pd with "Cf" for $\mathrm{N}=512, \mathrm{SNR}=-15 \mathrm{~dB}$ with $\mathrm{K}=2,5$

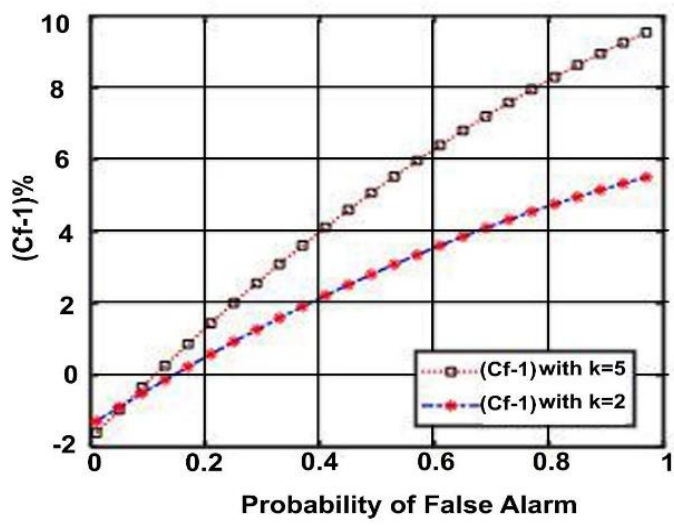

Figure 4. Deviation error $\%$ of the simulated test statistic $(\varepsilon)$ for $\mathrm{N}=512, \mathrm{SNR}=-15 \mathrm{~dB}$ with $\mathrm{K}=2,5$

\section{SIMULATION DISCUSSION}

This section presents and discusses the results obtained from intensive computer simulations and compares them with the theoretical results obtained from the proposed analytical study. These comparisons have included many important aspects and most of the factors that affect the accuracy of the suggested detection methodology in CR networks as listed in Table 1. Also, the accuracy and verification of the proposed theoretical study were confirmed by several comparisons with one of the previous but reliable studies [20]. The efficiency of the proposed sensing methodology has been ascertained by comparing it with the energy detection method [24, 25] and ensuring that the obtained results are identical to the previous results which are reported in the paper. The mean and variance of the proposed analytical formulas for two hypotheses $\mathrm{H}_{0}$ and $\mathrm{H}_{1}$ have been verified by comparing the final computer-simulated results with the analytical results under different simulation parameters as depicted in Figures 5 and 6 . It is enough to verify the mean and variance of MTM technique under the hypothesis $\mathrm{H}_{1}$.

Table 1. Simulation parameters

\begin{tabular}{ccccc}
\hline Sample $(\mathrm{N})$ & SNR $(\mathrm{dB})$ & Taper $(\mathrm{K})$ & Pf & Figure \\
\hline $512 \& 256$ & {$[-15$ to 15$]$} & $5 \& 2$ & --- & 5 \\
$512 \& 256$ & {$[-20$ to 0$]$} & $5 \& 2$ & --- & 6 \\
$512 \& 256$ & -10 & {$[2$ to 20$]$} & --- & 7 \\
512 & $-10 \&-15$ & $5 \& 2$ & {$[0$ to 1$]$} & $8 \& 11 \& 12$ \\
512 & {$[-25$ to 0$]$} & $5 \& 2$ & 0.1 & 9 \\
{$[500$ to 5000$]$} & -15 & $5 \& 2$ & 0.1 & $10 \& 13$ \\
\hline
\end{tabular}

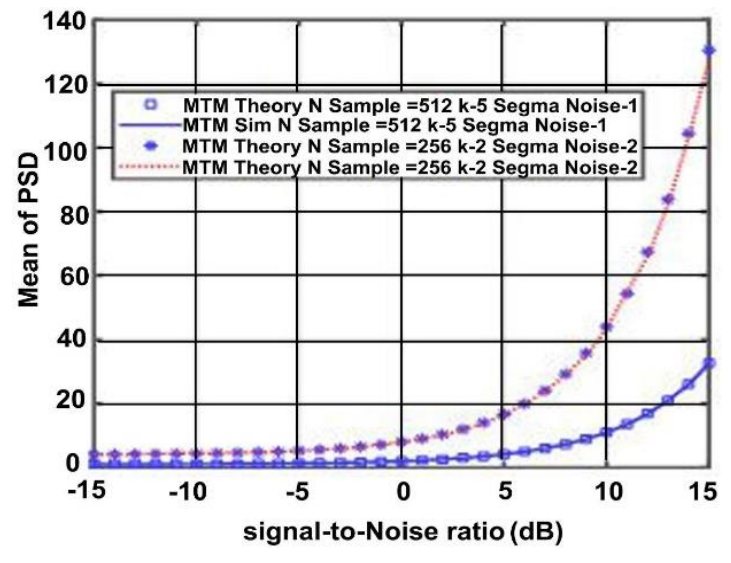

Figure 5. Verification of proposed analytical mean formula under hypothesis $\mathrm{H}_{1}$ with different simulation parameters

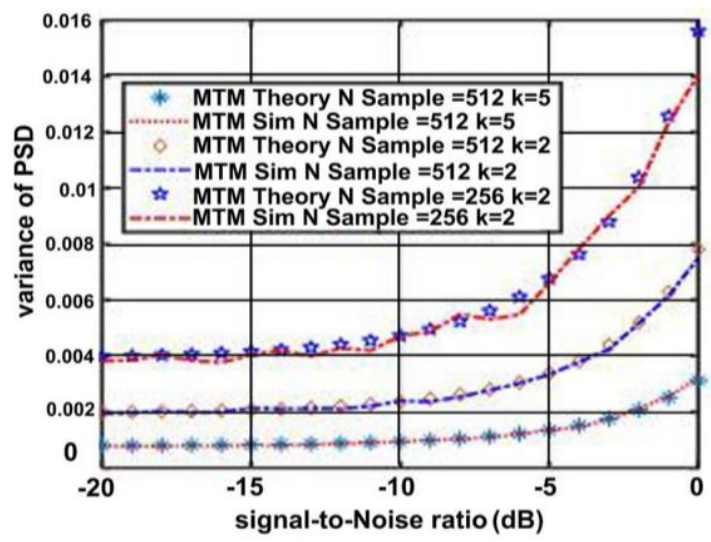

Figure 6. Verification of proposed analytical variance formula under hypothesis $\mathrm{H} 1$ with different simulation parameters 
Figure 5 shows the theoretical mean of MTM technique under hypothesis $\mathrm{H}_{1}$, which is given by (19), along with the simulated one, which is computed according to the first method, versus the signal-tonoise ratio. Obviously, the mean increases as SNR increases, which achieves he general concept of the estimator. Figure 6 shows the theoretical variance of MTM technique under hypothesis $\mathrm{H}_{1}$, given by (21), along with the simulated one computed according to the first method, versus SNR. Figure 7 depicts the theoretical variance of MTM technique under hypothesis $\mathrm{H}_{1}$, along with the simulated one, versus the number of tapers "K" under different simulation conditions. The Figure shows that the proposed formula obeys the MTM theoretical theory, where, the variance is a decreasing function with increasing K.

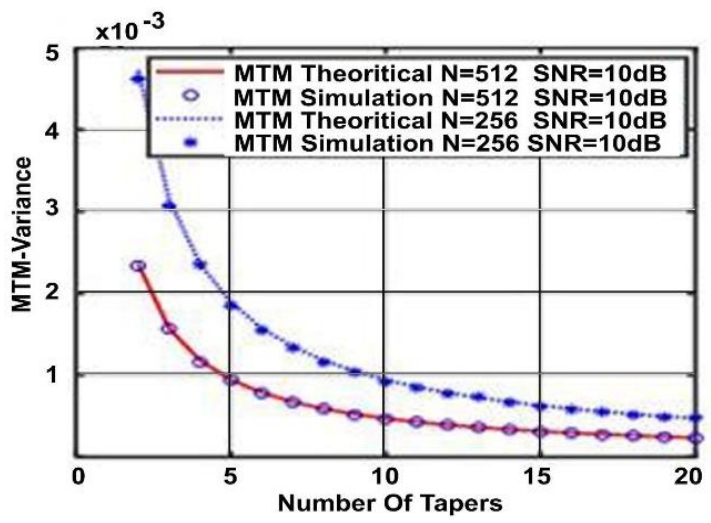

Figure 7. Verification of proposed analytical variance formula under hypothesis H1 versus tapers (K)

From the above three Figures 5, 6, and 7, it is obvious that the proposed analytical formulas for the mean and the variance under hypothesis $\mathrm{H}_{1}$ are accurate and they are valid to represent the real MTM system under different simulation conditions. Consequently, the proposed analytical formulas under hypothesis $\mathrm{H}_{0}$ are accurate and they are valid to represent the real MTM system under different simulation conditions. The Results are not similar to the mean and variance mentioned in [22] where the variance does not change with $\mathrm{K}$ and mean changes with $\mathrm{K}$. This means that the mean and variance contradict the concept of MTM.

The receiver operating characteristics (ROC) curve considers the key measurement metric that is used to analyze and evaluate the overall detection rate of spectrum sensing techniques. Therefore, it is used to verify the proposed analytical formulas of $\mathrm{P}_{\mathrm{d}}, \mathrm{P}_{\mathrm{f}}$, and $\gamma$ as a first verification scenario. Figure 8 illustrates the accuracy of the relationship between probability of false alarm and probability of detection which has been verified by comparing theoretical ROC curves determined by (22) and (23) with that one derived directly from computer-generated data using (10) and (11), i.e., according to the first method.

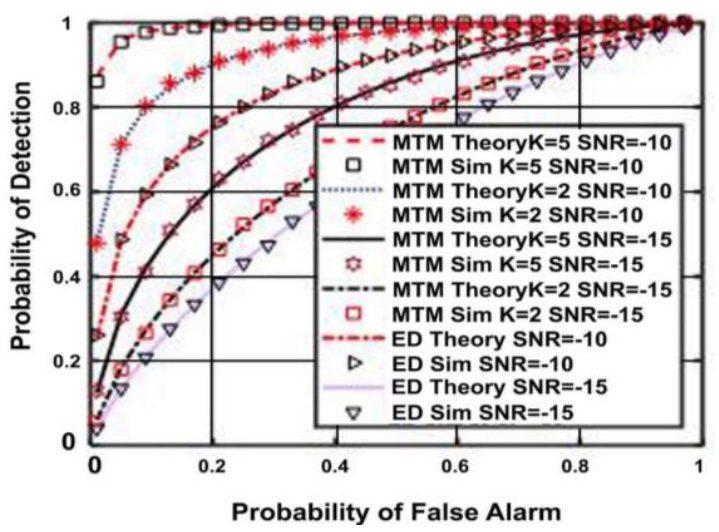

Figure 8. Probability of detection versus probability of false alarm (ROC curves) for MTM and ED with $\mathrm{N}=512$, and different values for SNR and $\mathrm{K}$

Under different simulation parameters such as $\mathrm{N}=512$ and different values for $\mathrm{SNR}$ and $\mathrm{K}$, the proposed paradigm was simulated. From Figure 8, the ROC which was generated from the proposed theoretical formulas for the probability of detection $\left(\mathrm{P}_{\mathrm{d}}^{\mathrm{MTM}}\right)$ and the probability of false alarm $\left(\mathrm{P}_{\mathrm{f}}^{\mathrm{MTM}}\right)$,

Verification analysis for the reliable analytical multi-taper detector in next... (Hebat Allah O. Selim) 
is identical to the one generated from the simulation model under a different set of false-alarm and simulation parameters. The results assert that the precision of the proposed theoretical formulas for the proposed analytical formulas of $\mathrm{P}_{\mathrm{d}}, \mathrm{P}_{\mathrm{f}}$, and $\gamma$ match well under all settings of false-alarm rate and other system parameters.

From Figure 8, it is obviously shown that, the probability of detection for MTM is almost 99\% with a probability of false alarm about $10 \%$ for $\mathrm{K}=5$ and $\mathrm{SNR}=-10 \mathrm{~dB}$. In the same figure, the detection rate of MTM is compared with Energy detection reported in [24]. We find that MTM exceeds Energy detection by about $40 \%$ and $20 \%$ at $P_{f}=0.1$ for $K=5$ and $K=2$, respectively, using the same parameters.

The effect of number of tapers $(K)$ on the relation between $P_{d}$ and SNR is verified by plotting the values of $\mathrm{Pd}$ for both theoretical and simulation results versus different SNR values with different number of tapers K, as shown in Figure 9. From Figure 9, it is obvious that the performance of detection is enhanced and the detector becomes more reliable with increasing "K" under the same SNR, and $\mathrm{P}_{\mathrm{f}}$. For example, at $P_{f}=0.1$ and $S N R=-15 d B$, the $P_{d}$ equals about $44 \%$ at $K=5$ while it equals about $29 \%$ at $K=2$. Also, it is noted that the proposed theoretical curve matches well with that generated by simulation under all settings of SNR and $\mathrm{K}$. The proposed paradigm verification and performance are tested for a wide range of $\mathrm{N}$ (500-5000) with different $\mathrm{K}(2,5)$, at $\mathrm{P}_{\mathrm{f}}=0.1$ and $\mathrm{SNR}=-15 \mathrm{~dB}$ and compared with $\mathrm{ED}$, as shown in Figure 10.

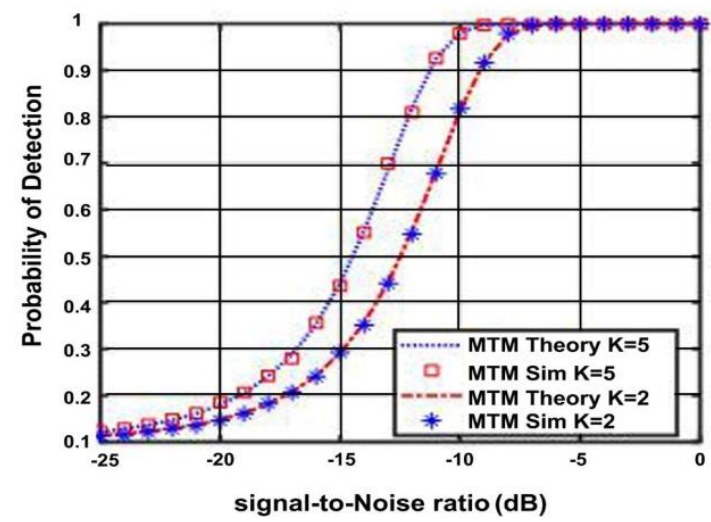

Figure 9. Probability of detection versus SNR at $\mathrm{Pf}=0.1$ and $\mathrm{N}=512$ for different tapers $\mathrm{K}$

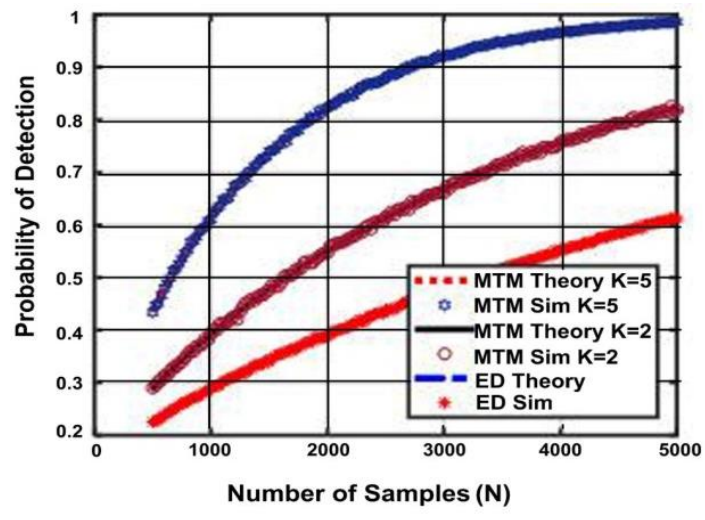

Figure 10. Probability of detection versus N at $\mathrm{Pf}=0.1$ and $\mathrm{SNR}=-15 \mathrm{~dB}$ for different number of tapers $\mathrm{K}$

From Figure 10, it is obvious that the detection rate is enhanced and the detector becomes more reliable with increasing " $\mathrm{N}$ " under the same SNR, and $\mathrm{P}_{\mathrm{f}}$. Also, For $\mathrm{N}=5000$, the maximum achievable $\mathrm{P}_{\mathrm{d}}$ for $\mathrm{K}=5$ is "0.986", for $\mathrm{K}=2$ is "0.819", and for ED is" 0.61 ". The number of samples which is necessary to achieve $\mathrm{P}_{\mathrm{d}}=0.819$ for $\mathrm{K}=5$ is " 1975 " obeys (30).

$$
\text { Required } N_{\text {for } K=K 1}=\frac{K 2}{K 1} \text { Required } N_{\text {for } K=K 2}
$$

Also, the samples size which is desired to obtain $P_{d}=0.61$ for $K=5$ is "1000" obeys (31).

$$
\frac{N_{M i n}^{E D}}{N_{M i n}^{M T M}}=K
$$

According to the second approach, in which the test statistic must be multiplied by "Cf" before making the decision to reduce the deviation error between the theoretical and simulation results. The proposed correction factor accuracy and reliability are verified for a wide range of $\mathrm{P}_{\mathrm{f}}$ with $\mathrm{K}=2$, 5, $\mathrm{SNR}=-15,-10 \mathrm{~dB}$, and $\mathrm{N}=512$, as shown in Figure 11. In the same Figure, the detection performance of MTM is compared with Energy detection. The same results illustrated in Figure 8 are obtained.

Moreover, the proposed paradigm is compared with the model reported in [20]. As depicted in Figure 12, the detection rate of the proposed paradigm achieves the same rate of the paradigm reported in [20] under different values of false-alarm rate, especially, in the low and moderate SNR ranges. It is worth mentioned that the proposed paradigm achieves the same detection performance as the model in [20], 
but is less computational demanding. Also in Figure 13, the required sample size for the proposed paradigm and the paradigm reported in [20] are simulated as a function of Tapers (K). It is clear that the proposed MTM model requires the same sample size as the model referenced in [20] to achieve the same detection performance as the model.

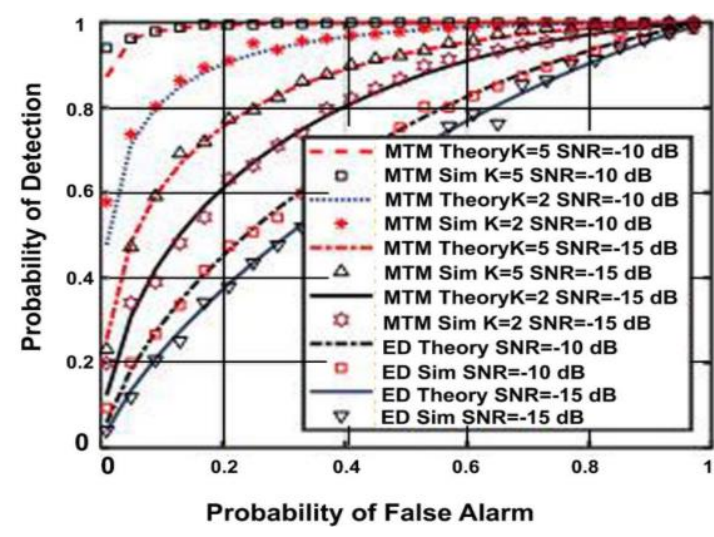

Figure 11. ROC curves for different simulation parameters generating using second method with the statistics are corrected using "Cf" at $\mathrm{N}=512$

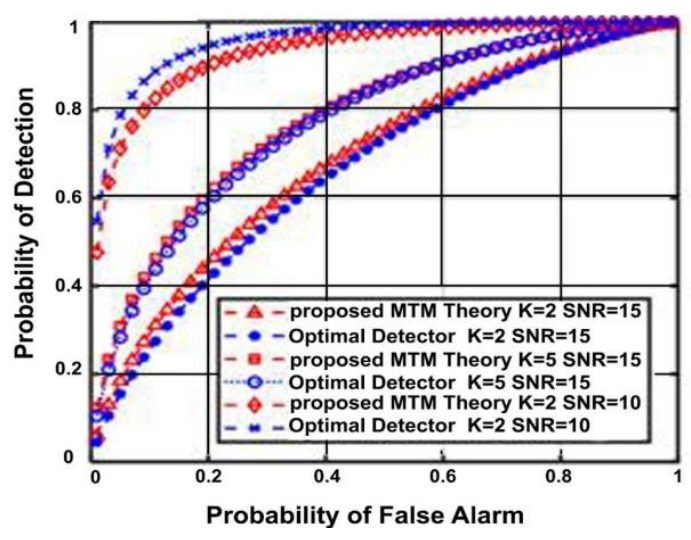

Figure 12. Comparison between ROC curves generated using the proposed paradigm and the paradigm reported in [20] with $\mathrm{N}=512$

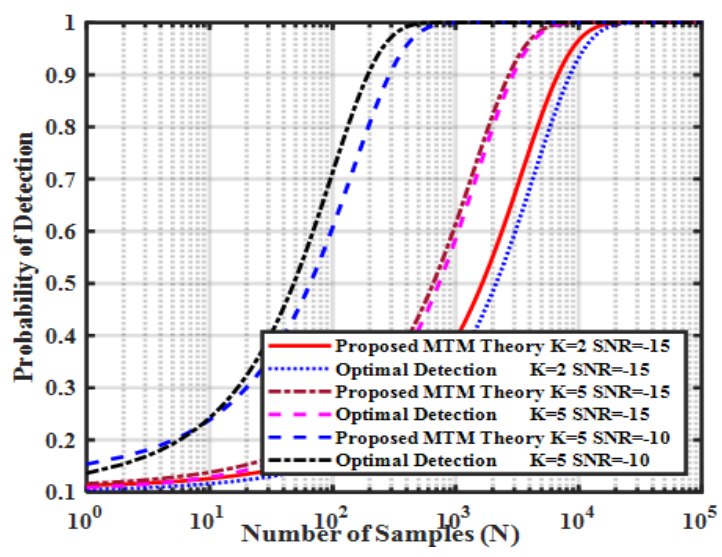

Figure 13. Performance comparison of the proposed paradigm with the paradigm reported in [20] with respect to the number of samples with $\mathrm{Pf}=0.1$

\section{CONCLUSION AND FUTURE WORK}

In this paper, a simple and computationally efficient analytical spectrum sensing method to discover the radio spectrum in CR is developed. MTM detector surpasses the detection rate of the energy detector by $40 \%$ given a fixed probability of false alarm $10 \%, \mathrm{~N}=512, \mathrm{SNR}=-15 \mathrm{~dB}$, and $\mathrm{K}=5$. Based on this work, some perspectives for future work are suggested. There is a need to investigate the practical implementation of the proposed analytical technique on a real-time test-bed, to determine correlation between simulation and implementation results.

\section{REFERENCES}

[1] J. Mitola and G. Q. Maguire, "Cognitive radio: making software radios more personal," IEEE Personal Communications, vol. 6, no. 4, pp. 13-18, Aug. 1999.

[2] P. E. Numan, K. M. Yusof, M. N. Marsono, S. K. S. Yusof, Mohd H. Mohd Fauzi, S. Nathaniel, E. N. Onwuka, and M. A. Baharudin, "On the latency and jitter evaluation of software defined networks," Bulletin of Electrical Engineering and Informatics, vol. 8, no. 4, pp. 1507-1516, 2019.

[3] O. A. Aboaba, "A review of signal parameter estimation techniques," American Association for Science and Technology (AASCIT) Communications, vol. 2, no. 2, pp. 25-28, 2015. 
[4] S. Lakhal and Z. Guennoun, "Equity-based free channels assignment for secondary users in a cognitive radio network," International Journal of Electrical \& Computer Engineering, vol. 9, no. 3, pp. 2057-2063, 2019.

[5] S. D. Borde and K. R. Joshi, "Enhanced signal detection slgorithm using trained neural network for cognitive radio receiver," International Journal of Electrical and Computer Engineering, vol. 9, no. 1, pp. 323-331, 2019.

[6] Deepa N. Reddy, and Y. Ravinder, "A statistical approach to spectrum sensing using bayes factor and p-values," International Journal of Electrical \& Computer Engineering, vol. 9, no. 4, pp. 2910-2917, 2019.

[7] M. K. Kaushik, Y. Yoganandam, and S. K. Sahoo, "Sensing and sharing schemes for spectral efficiency of cognitive radios," International Journal of Electrical and Computer Engineering, vol. 8, no. 5, pp. 2934-2941, 2018.

[8] M. Tuberquia and C. Hernandez, "New approaches in cognitive radios using evolutionary algorithms," International Journal of Electrical and Computer Engineering, vol. 8, no. 3, pp.1636-1646, June 2018.

[9] S. Atapattu, C. Tellambura and H. Jiang, "Energy detection based cooperative spectrum sensing in cognitive radio networks," IEEE Transactions on Wireless Communications, vol. 10, no. 4, pp. 1232-1241, April 2011.

[10] K. Arshad and K. Moessner, "Robust spectrum sensing based on statistical tests," IET Communications, vol. 7, no. 9, pp. 808-817, June 2013.

[11] P. Stoica and T. Sundin, "On nonparametric spectral estimation," Circuits, Systems and Signal Processing, vol 18, pp 169-181, 1999.

[12] W. Wang, "Spectrum sensing for cognitive radio," 2009 Third International Symposium on Intelligent Information Technology Application Workshops, Nanchang, pp. 410-412, 2009.

[13] X. Zhang, R. Chai, and F. Gao, "Matched filter based spectrum sensing and power level detection for cognitive radio network," 2014 IEEE Global Conference on Signal and Information Processing (GlobalSIP), Atlanta, GA, pp. 1267-1270, 2014.

[14] A. Wilfred and Okonkwo O. R., "A review of cyclostationary feature detection based spectrum sensing technique in cognitive radio networks," E3 Journal of Scientific Research, vol. 4, no. 3, pp. 041-047, Sept. 2016.

[15] D. Damodaram and T. Venkateswarlu, "Efficient hardware architecture for cyclostationary detector," Bulletin of Electrical Engineering and Informatics, vol. 5, no. 3, pp. 340-346, 2016.

[16] Y. Liang, Y. Zeng, E. C. Y. Peh and A. T. Hoang, "Sensing-throughput tradeoff for cognitive radio networks," IEEE Transactions on Wireless Communications, vol. 7, no. 4, pp. 1326-1337, April 2008.

[17] S. Haykin, "Cognitive radio: Brain-empowered wireless communications," IEEE Journal on Selected Areas in Communications, vol. 23, no. 2, pp. 201-220, Feb. 2005.

[18] D. J. Thomson, "Spectrum estimation and harmonic analysis," Procdings of the IEEE, vol. 70, no. 9, pp. 1055-1096, Sept. 1982.

[19] M. K. Jataprolu, R. D. Koilpillai, and S. Bhashyam, "Optimal MTM spectral estimation based detection for cognitive radio in HDTV," 2012 National Conference on Communications (NCC), Kharagpur, pp. 1-5, 2012.

[20] T. Chiang, J. Lin, and H. Ma, "Optimal detector for multitaper spectrum estimator in cognitive radios," GLOBECOM 2009-2009 IEEE Global Telecommunications Conference, Honolulu, pp. 1-6, 2009.

[21] Y. Xin, K. Kim, and S. Rangarajan, "Multitaper spectrum sensing of OFDMA signals in frequency selective fading environment," 2011 6th International ICST Conference on Cognitive Radio Oriented Wireless Networks and Communications (CROWNCOM), Osaka, pp. 56-60, 2011.

[22] A. A. Owayed, Z. A. Mohammed, and A. A. Mosa, "Probabilities of detection and false alarm in multitaper based spectrum sensing for cognitive radio systems in AWGN," 2010 IEEE International Conference on Communication Systems, Singapore, 2010, pp. 579-584.

[23] S. Kay, "Fundamentals of statistical signal processing-Detection theory," Signal Processing Series, Prentice Hall, vol. 2, 1998.

[24] M. Z. Alom, T. K. Godder, M. N. Morshed, and A. Maali, "Enhanced spectrum sensing based on energy detection in cognitive radio network using adaptive threshold," 2017 International Conference on Networking, Systems and Security (NSysS), Dhaka, pp. 138-143, 2017.

[25] S. Atapattu, C. Tellambura, and H. Jiang, "Energy detection for spectrum sensing in cognitive radio," Springer Briefs in Computer Science, Springer, Chapter 2, 2014. 